\title{
Technologies To Improve The Decision-Making Process Of Real Estate Appraisers: XML, Intelligent Agents, Avms, And Web Services
}

Timothy H. Greer, (Email: tgreer@mtsu.edu), Middle Tennessee State University Mirza B. Murtaza, (Email: mmurtaza@mtsu.edu), Middle Tennessee State University

\begin{abstract}
Pressure to expedite mortgage originations has prompted the need to accelerate the appraisal process for residential real estate. The appraisal aspect of a mortgage origination is one area where decision support can play a major role in redefining the process. This study presents propositions that incorporate new technologies into the decision-making process of a residential real estate appraiser. The four propositions deal with intelligent agents, XML, automated valuation models (AVMs), and web services. Intelligent agents have the ability to redefine how appraisal firms create, maintain, and update their databases. XML offers enhanced data handling capabilities, while automated valuation models offer the ability to drastically reduce the time required to render an estimate of value. These two technologies can be combined with web services to make the real estate appraisal application available anywhere and anytime of the day.
\end{abstract}

\subsection{Introduction}

$M$

any industries have encountered technological innovations that have influenced the business environment in which they operate. Laws, regulation, and industry standards may be inhibiting factors impacting the adoption of new technological developments in the appraisal industry. However, the regulating bodies are pushing for a more expeditious appraisal process. Increased use of technology would be one possible way to accelerate the appraisal process. Automated valuation models (AVMs) utilize current software programs to enhance the appraisal process. AVMs are currently based on methodologies ranging from multiple regression analysis to complex expert systems and neural networks. One area of concern with these methodologies is the need for a large data sample. This is especially problematic when dealing with residential real estate appraisal. While several organizations offer residential real estate data for sale, coverage is somewhat segmented and limited. Another limiting factor is incomplete data, or the inability to obtain data on demographics and neighborhood characteristics. Since large amounts of data are now published on the Internet, a potential solution to the data problem is the use of intelligent agents. The benefit of intelligent agents in the appraisal process could be significantly increased if XML was adopted by the appraisal and real estate industries. The purpose of this study is to propose the use of new decision-making technologies in the residential real estate appraisal process. The underlying purpose of integrating new technology into the appraisal process is to provide fast and improved decisions. The improved decisions could be attributed to more pertinent data, better data quality and a more efficient means to complete the appraisal assignment. Three important facets of the residential real estate appraisal process are acquiring data on comparable properties, maintaining data on comparable properties, and rendering

Readers with comments or questions are encouraged to contact the authors via email. 
estimates of value. The following discussion describes how intelligent agents, XML, automated valuation models, and web services could be used to enhance these facets of the residential real estate appraisal process.

This paper is structured as follows. The following section provides an overview of issues concerning data and the Internet. Section 3 presents an example of how XML could be incorporated and used by the appraisal industry. Section 4 of the paper discusses AVM methodologies, focusing specially on expert systems and neural networks. Section 5 presents a brief overview of web services and how the technologies outlined in this paper can be used to provide web services for real estate appraisal. The last section provides a summary of the study and what possible implications the use of new technology will have on the decision-making process of the residential appraiser.

\subsection{Intelligent Agents}

Proposition 1: Use intelligent agents to help build, maintain, and update the database of comparable properties.

One of the most popular methods of rendering an estimate of value is to compare the subject property with comparable or like properties. This method is known as the market value approach. This approach requires the appraiser to maintain a database of properties. The database needs to reflect the relevant market conditions for each appraisal assignment. Therefore in some cases the database may need to be continuously updated and in others it may require infrequent changes, depending on market conditions. The process of conducting an appraisal could benefit in many ways from automation or recent technological advances. Intelligent agents have the potential to redefine the appraisal process. One way is through the creation and maintenance of a database of comparable properties. This application would be ideal for an intelligent agent. An intelligent agent could be assigned to search the Internet or an organization's Intranet. An intelligent agent has the ability to continuously search the Internet for information on predefined property, neighborhoods, or geographic regions. The asking price, assessment value, crime statistics, educational characteristics or school quality, and other viable information used in the valuation of residential property could also be obtained by an intelligent agent. Numerous sites exist that an agent could visit; currently there are US national, regional, and local multiple listing services that provide property characteristics and asking price ${ }^{1}$. Several sites now list the actual sales price of individual properties ${ }^{2}$. If an appraisal firm wished to enhance their database by adding crime statistics and demographic characteristics an intelligent agent could easily perform this task. While the census is conducted every decade other sources are available for such information. In the United States, The US Department of Justice, Federal Bureau of Investigation, and state police agencies all report crime statistics on the Internet.

Figure 1 depicts the basic architecture of an intelligent agent. An agent has both transient or current information and long-term knowledge and data. The heart of the intelligent agent is a controller that can be given specific behavioral characteristics depending upon its job. The intelligent agent performs tasks or actions that can be both declarative and procedural as needed. There can be various types of reasoning strategies employed by the agent, e.g., case-based, and its capabilities depend upon the set of tasks it can perform and its reasoning methods. To complete its tasks, it must have a collection of "how to" procedures that are represented in various forms, such as rules, knowledge bases (rule sets), or methods.

The potential uses for intelligent agents in the appraisal process are numerous. Therefore the incorporation of a community of intelligent agents would seem plausible. Each agent however would need to be able to carry out its assigned duties independent of other agents [HAY 99]. In this case it would be part of a community of agents that would be responsible for maintaining a repository of data used in the appraisal process. Agents may or may not communicate with each other, depending upon the goals of the organization. Agents that communicate with each other need to have some type of communication protocol established [BRA 99]. Some agents may compete against each other; therefore communication between some agents may not be necessary. One possible goal of having

\footnotetext{
${ }^{1}$ http://www.realtor.com

${ }^{2}$ http://www.tnrealestate.com
} 
agents competing against each other would be to verify information and improve the quality of the database. The number of agents would depend upon the organization and the objective assigned to the community of agents.

There are a few issues that should be considered. Agent vulnerability, sabotage and mis-information are all issues that should be addressed. Many sites will not allow agents to enter or they create obstacles that inhibit the agent from completing its assigned task. The construction of a firewall or requiring the user/agent to login hinders the agent from task completion. Avoiding sites that have firewalls and obtaining approval from sites prior to the agent visiting the site would help the agent achieve its goal. These steps will help alleviate many of the barriers facing intelligent agents.

The term agent has had the attention of the artificial intelligence community for the past decade. One possible reason for their slow adoption into the business process of many industries could be attributed to the lack of tools. The overall lack of graphical user interfaces (GUI) for agent tools and a high learning curve for many of the available tools have limited the use of agents [BRA 99]. However, businesses will undoubtedly be very interested in any process or technology that could help them achieve a competitive advantage.

Information extraction is a key to using intelligent agents to extract information from the Internet [KUS 99]. Most documents on the Internet are HTML (Hyper-Text Markup Language) documents. HTML is the universal markup language of the Internet. HTML allows a developer the ability to present text and multimedia on the Internet. HTML is not concerned with the actual data being displayed but only the presentation aspect of the document. However, XML is a markup language that focuses on the data. This study proposes that XML would increase the efficiency of an intelligent agent as well as the overall data handling capability of the appraisal organization.

Figure 1: Architecture of an Intelligent Agent

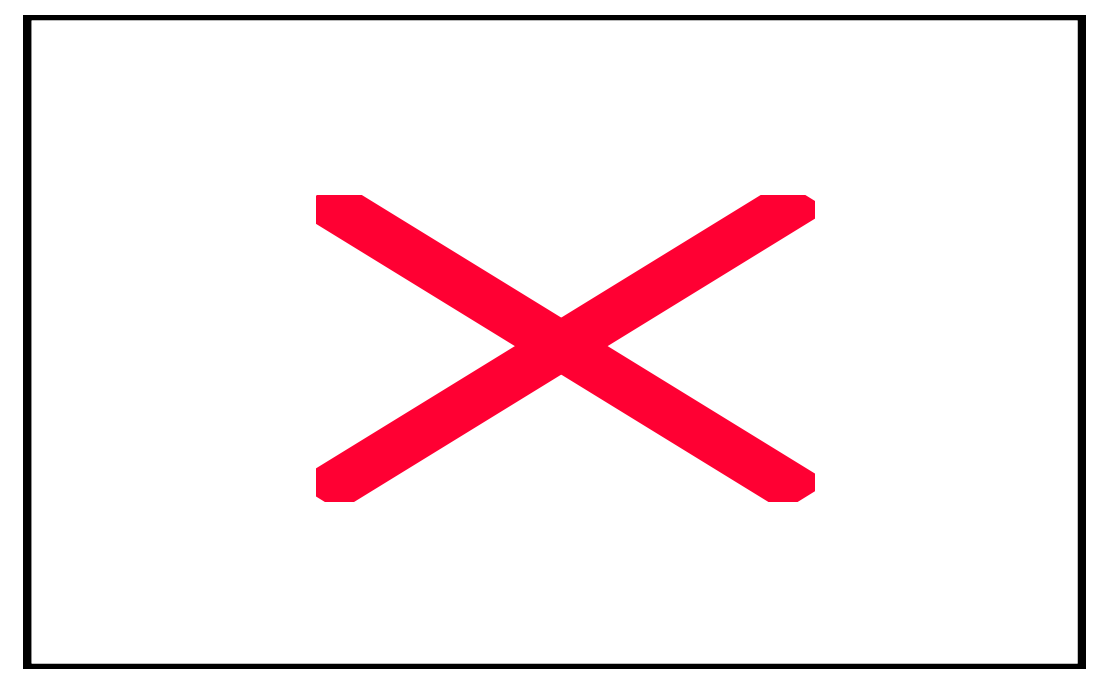

\subsection{XML}

Proposition 2: Use XML with intelligent agents to help build, maintain, and update the database of properties.

The decision-making process of the residential real estate appraiser is highly dependant on data. XML gives meaning to data and would increase the value of the data repository. Incorporating XML could enhance the quality of the data repository. Issues such as data availability, incomplete data, and outdated data could be addressed 
through the use of XML and intelligent agents. There are several web sites that offer nationwide property listings for the United States. Most of the sites query their database based on several pieces of information provided by the user. The first series of questions usually addresses the location of the property, which may start at the state level and progress to a specific section of a township or county. Once the location is decided upon several other questions are presented to the user. The questions may include price range; maximum and minimum square footage and number of bedrooms. The table below shows the potential results or listings of a sample query from an on-line Multiple Listing Service (MLS).

Table 1: Sample results From an On-line MLS

\begin{tabular}{|l|l|c|c|c|c|c|c|c|c|}
\hline \multicolumn{1}{|c|}{ MLS } & \multicolumn{1}{|c|}{ Address } & $\begin{array}{c}\text { Square } \\
\text { Footage }\end{array}$ & Bed & Bath & $\begin{array}{c}\text { Half } \\
\text { Baths }\end{array}$ & Stories & $\begin{array}{c}\text { Parking } \\
\text { Spaces }\end{array}$ & $\begin{array}{c}\text { Fire- } \\
\text { places }\end{array}$ & \multicolumn{1}{c|}{ Price } \\
\hline 277727 & 232 Mission Ridge & 2200 & 4 & 2 & 2 & 2 & 2 & 2 & $\$ 151,900$ \\
\hline 992921 & 125 Forge Ridge & 2450 & 3 & 2 & 2 & 2 & 2 & 2 & $\$ 155,000$ \\
\hline 893944 & 113 Biltmore & 2000 & 4 & 2 & 2 & 2 & 2 & 2 & $\$ 155,900$ \\
\hline 783557 & 180 Forge Ridge & 2150 & 3 & 2 & 2 & 2 & 2 & 2 & $\$ 156,000$ \\
\hline
\end{tabular}

Notice that the number of full bathrooms, half bathrooms, fireplaces, stories, and parking spaces are the same for each property ("2"). The significance here is demonstrated in the html code below. There are several instances where the number " 2 " is displayed between the HTML tags. In an attempt to simplify the code, the html code below does not contain any formatting. Most sites today incorporate some type formatting or style sheet to enhance the presentation of their web pages. This only increases the effort required to obtain information from the data presented. The difficulty in establishing an association with each column heading and a corresponding "2" signifies the need for the adoption of XML.

Table 2: Sample HTML Code:

\begin{tabular}{|l|}
\hline HTML Code \\
\hline$<\mathrm{HTML}>$ \\
$\ldots .<\mathrm{tr}>$ \\
$<\mathrm{td}>277727</ \mathrm{td}>$ \\
$<\mathrm{td}>232$ Mission Ridge $</ \mathrm{td}>$ \\
$<\mathrm{td}>2200</ \mathrm{td}>$ \\
$<\mathrm{td}>4</ \mathrm{td}>$ \\
$<\mathrm{td}>2</ \mathrm{td}>$ \\
$<\mathrm{td}>2</ \mathrm{td}>$ \\
$<\mathrm{td}>2</ \mathrm{td}>$ \\
$<\mathrm{td}>2</ \mathrm{td}>$ \\
$<\mathrm{td}>\$ 151,900</ \mathrm{td}>$ \\
$</ \mathrm{tr}>\ldots \ldots \ldots \ldots . \mathrm{HTML}>$ \\
\hline
\end{tabular}

XML is derived from SGML, or the Standardized Markup Language. HTML is widely used to display data; it is a markup language to present information on a web page. XML allows an organization or industry to create tags to mark up documents. XML can be used to describe, store, and exchange data. A key advantage of $\mathrm{XML}$ is that it can be used to structure documents as well as disseminate information. Unlike HTML where the presentation and the content are intertwined, XML separates the content. XML is capable of displaying residential property information in a readable format for both machines and humans. XML tags convey the meaning of the data, which enhances functionality [GLU 99]. Therefore in our example the machine as well as the browser can 
easily decipher and interpret the content of an XML document. Figure 2 uses Microsoft's XML notepad and the built in XML parser of Internet Explorer 5 to display a property listing.

Figure 2: XML Property Listing:

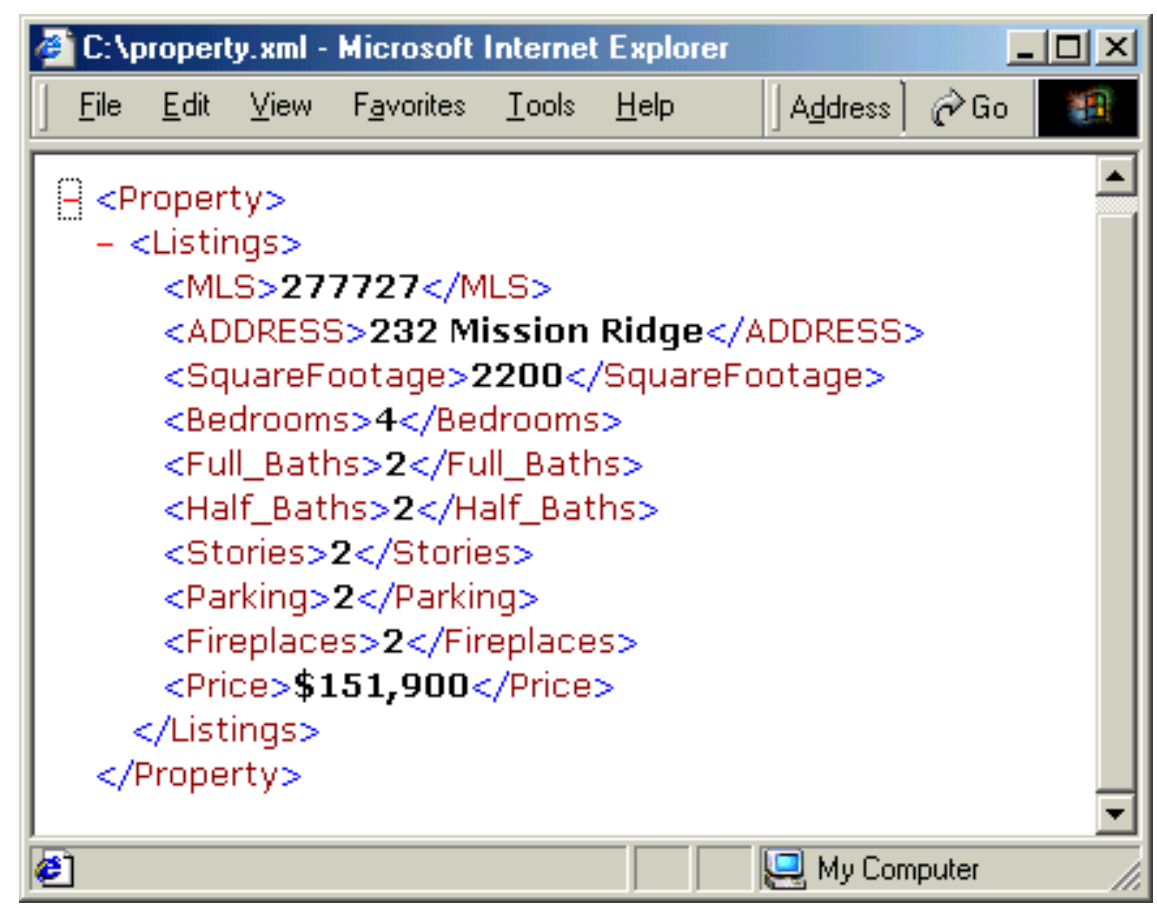

While the above figure displays the XML tags, the use of a style sheet could be used to display the document is a more browser friendly format. The use of XML facilitates the machine readability of a document. Ideally the adoption of XML tags would be industry wide. Even if the tags vary, the above Figure should indicate the potential power of XML to structure data. In comparing Figure 2 and Table 1, the XML code and the HTML code for the same property, it should be clear that XML offers more structure and meaning to a document than HTML. This paper proposes that the use of XML would significantly increase the efficiency of intelligent agents in the appraisal process. While agents could be assigned the duty of updating, maintaining, and generating data for the database of properties, automated valuation models could help in the process of rendering an estimate of value.

\subsection{Automated Valuation Models: AVMs}

Proposition 3: Use Automated Valuation Models to help render an estimate of value in the appraisal process.

The economic expansion of the 1990s has fueled the real estate industry. Forces in the real estate industry are pushing for the process to become more expedient. Every residential real estate sales transaction involving a financial institution relies on a residential real estate appraisal. A potential tool that would allow appraisers to operate more efficiently is an AVM. Automated valuation models (AVMs) can drastically reduce the amount of time required to perform an appraisal. When rendering an estimate of value an AVM may use anything from a simple statistical operation to a genetic algorithm. The AVM is designed to assist the appraiser in rendering an estimate of value; the AVM is not a self-contained appraisal generating mechanism. Two popular methodologies used in AVMs are neural networks and expert systems. 


\subsection{Neural Networks:}

Although the theory underlying artificial neural networks was developed in the 1940s and 1950s, advances in technology have made applications such as valuing residential real estate possible using a neural network. Artificial neural networks are mathematical models of the human brain. A feedforward neural network is a widely used network topology. The feedforward multi-layered network relies on a set of corresponding inputs and output in order to train the network. The input value is multiplied by a weight value. The multiplied result is sent to the hidden node at the end of the connection. Initially the weights are randomly set based on a uniform distribution. The weights will change as the network is trained. Numerous algorithms have been developed that assign a weight value to each connection. The multiplied results of the input value and the weight value are sent through an activation function. The most common activation function is the sigmoid function.

The sigmoid function is a cumulative distribution function that produces an output. The value of each hidden node is multiplied by additional weight connections. This result is the output of the network. The network converges when the trained network produces output within a desired range of the actual output.

Misconceptions concerning neural networks usually can be traced to the hidden layer. A "black box" is undoubtedly a term most people would associate with a neural network. The hidden layer is usually targeted as the black box. The weights of the network are usually housed in a weight matrix. This weight matrix allows for an analytical expression to be derived. For instance if a network has 3 input nodes and 2 hidden nodes, the weight matrix would have 6 components. Each hidden node is assigned an output from the activation function. While the weight matrix may change as the network trains, once convergence is reached a weight matrix exists that allows for mathematical scrutiny. Therefore, the notion of neural networks as black boxes can be disputed. One point of caution concerning the hidden layer, too many hidden nodes can create over parameterization. This is where the network memorizes a specific set of input/output combinations. Memorization by the network may cause the network to blunder when tested out of sample.

Many algorithms are available to train a neural network. One of the most common training algorithms associated with neural networks is the backpropagation algorithm. The backpropagation algorithm is popular due to its simplicity. One can think of backpropagation as a simple hill climbing technique. When searching for optima, the backpropagation algorithm converges when the derivative of the function is zero or approaches zero. In order to find the optima the search is initialized and presented with a set of inputs, which are propagated through the network layers until output is reached. The difference between the actual output and the network output, the error term, is propagated back through the layers of the network, hence the name back propagation.

Even though backpropagation is widely used and is a relatively simple algorithm to use, it does have some disadvantages. The tendency of getting trapped in local optima is the most recognizable disadvantage of backpropagation. Other disadvantages include temporal instability, network paralysis, and the requirement of needing a differentiable function. These disadvantages have lead researchers to search for other training algorithms. The genetic algorithm unlike the backpropagation algorithm does not require a differentiable function.

The genetic algorithm was chosen because of its performance in past studies. The genetic algorithm is a search technique that mimics the natural order of selection. The genetic algorithm has been used to solve a number of different problems [HOL 92]. The popularity of the genetic algorithm is commonly attributed to the fact that it is a global search technique. The objective function does not have to be differentiable, which is another advantage associated with the genetic algorithm. There are a couple of limitations or disadvantages associated with the genetic algorithm. The objective function may need to be evaluated many times with the genetic algorithm, thus requiring vast amounts of processor time [DOR 95]. They realize however that with the rapid advances in computer technology this will probably only be a temporary limitation. Another limitation associated with the genetic algorithm is that the genetic algorithm may find a point close to the optima, but not exactly where the gradient is 
equal to zero. A hill climbing technique can be used to speed convergence once the genetic algorithm finds the global optima neighborhood [DOR 95].

The genetic algorithm provides an ideal algorithm to aid the residential property appraiser in finding and valuing property. The ability of the neural network to learn relationships between inputs and outputs along with its ability to map non-linear functions should prove to be a valued asset to the residential property appraiser. An important disadvantage to residential property appraisers is that the reasoning process is not easily obtained. If a question about a particular appraisal arises, the appraiser may feel uneasy trying to explain the inner workings of the neural network. It is due to this that an appraiser may experience improved results using an expert system.

Several researchers have used neural networks to estimate the value of property. They found that the neural network was able to outperform regression analysis [DO 92], [EVA 92], and [TAY 91]. While these results show promise, some researchers caution their use because of inconsistent results and the difficulties associated with using them [WOR 95]. Expert systems are one alternative to overcome the inconsistent results associated with a neural network.

\subsection{Expert Systems}

Another popular methodology that can be used in AVMs is an expert system. Expert systems offer many advantages to the appraiser. Expert systems produce consistent solutions and allow for the reasoning behind the solution to be easily traced. The expert system incorporates a series of rules or if-then statements, which provides the premises for the solution. The answers to the if-then statements are analyzed in conjunction with the knowledge base to produce the solution(s). Expert systems seem to provide an excellent means of selecting comparable properties. The knowledge base contains information on what an expert appraiser analyzes when they select their comparable properties.

Expert systems are designed to capture the knowledge of an expert in a particular field and store that knowledge in a computer. Then anyone could use the information to respond to an issue in that field with the knowledge of an expert by consulting the expert system. The end user could tap the expert's knowledge through a heuristic methodology. A limitation is that acquiring this knowledge to build and maintain the expert system is time consuming and expensive. Generally expert systems are built to solve a specific problem or are designed for a specific application. Expert systems are most effective when they are presented with highly structured problems.

Expert systems are dependent on storage facilities and detailed instructions, whereas neural networks are not. One advantage expert systems have over neural networks is the ability to easily trace the reasoning of the decision-making process notes [TAF 92]. However, the expert system does not have the ability to learn and uses no intuition in the decision making process. The ability to easily trace the decision making process is a major advantage concerning residential appraisals. If the estimate is challenged the first question usually asked is what were the comparable properties? The interested parties are also concerned with the characteristics of the comparable properties. The incorporation of expert systems allows for easy display of the comparable properties and their characteristics. Table 3 lists the advantages and disadvantages associated with expert systems.

Table 3: Advantages \& Disadvantages of Expert Systems

\begin{tabular}{|c|c|}
\hline ADVANTAGES & DISADVANTAGES \\
\hline Prediction of a range of solutions & Knowledge acquisition bottleneck \\
\hline Reasoning can be traced & Lacks learning ability/Uses no intuition \\
\hline More consistent solutions & Needs highly structured problem \\
\hline Applies a higher level of logic & Time consuming to build/Maintain \\
\hline
\end{tabular}


The ability of the expert system to generate consistent solutions would favor the appraiser trying to explain the model and how they arrived at a particular value. Through the use of the knowledge base the appraiser is able to apply a higher level of logic to the particular appraisal assignment.

Rendering an estimate of value is a fundamental task of the residential real estate appraiser. The residential real estate appraiser should be aware that the neural networks and the expert system offer many advantages. These advantages would allow the appraiser to make better decisions in regards to rendering an estimate of value. Whether the AVM uses an expert system or is based on a neural network, the output of the AVM should increase the effectiveness and efficiency of the appraiser.

While an appraiser is not forced to accept and implement these or any other technological innovations they may be at a competitive disadvantage if they continue to resist these developments. The use of an intelligent agent, XML, or an AVM depends on the circumstances associated with each individual appraisal assignment. Each assignment requires an appraiser to determine when an intelligent agent, XML, or an AVM may be appropriate. One underlying premise of this study is to publicize the benefits of incorporating intelligent agents, XML, and AVMs into the appraisal process.

\subsection{Web Services For Real Estate Appraisals}

Proposition 4: Use Automated Valuation Models to determine and Web Services to provide an estimate of value in the appraisal process anytime, anywhere.

Web services are a recent phenomenon that has received a lot of attention. Web services employ a distributed computing architecture that is made up of many different computers that communicate over the familiar network, the Internet, to form one system. The communication standards and protocols used in web services must be universally accepted - some of those are still emerging. In general, a web service is simply an application that exposes a web-accessible program interface. This service can be requested by client applications - web-based, windows or mobile devices. However, an additional requirement is that application data and format must be such that it can be understood by all kinds of client applications ( $\mathrm{SHO} 03$ ).

XML (Extensible Markup Language) is the basic format for representing data on a web services platform. Additionally, at a minimum, there are three components required for web services:

- $\quad$ The Simple Object Access Protocol (SOAP) provides the standard mechanism used for invoking or calling Web services.

- The Web Service Description Language (WSDL) is an XML-based grammar that describes web services, their functions, parameters, and return values to their potential consumers (SHO 03).

- $\quad$ The Universal Description, Discovery, and Integration (UDDI) is a standard for describing available web services components, which allows businesses to register with an Internet directory (like the yellow pages) that will help them advertise their services, so that companies can find one another and conduct transactions over the web.

Figure 3 describes the basic Web services model. All data exchange is performed using XML format over the World Wide Web's hypertext transport protocol (HTTP).

All the parties involved in real estate transactions can greatly benefit if there is a web service available for residential real estate appraisal. A real estate appraiser can access the same information whether at home, office or anywhere else and at any time of the day. Since the client service can run on office PC, laptop or mobile device. In such a web service, an AVM application would run on the server while all the data input and output will be exchanged in XML format. This would provide an integration of a traditional application with various types of Internet-based systems, the application would be exposed to the Internet as a Web service as shown in Figure 4. 
Figure 3: Basic Web Services Model

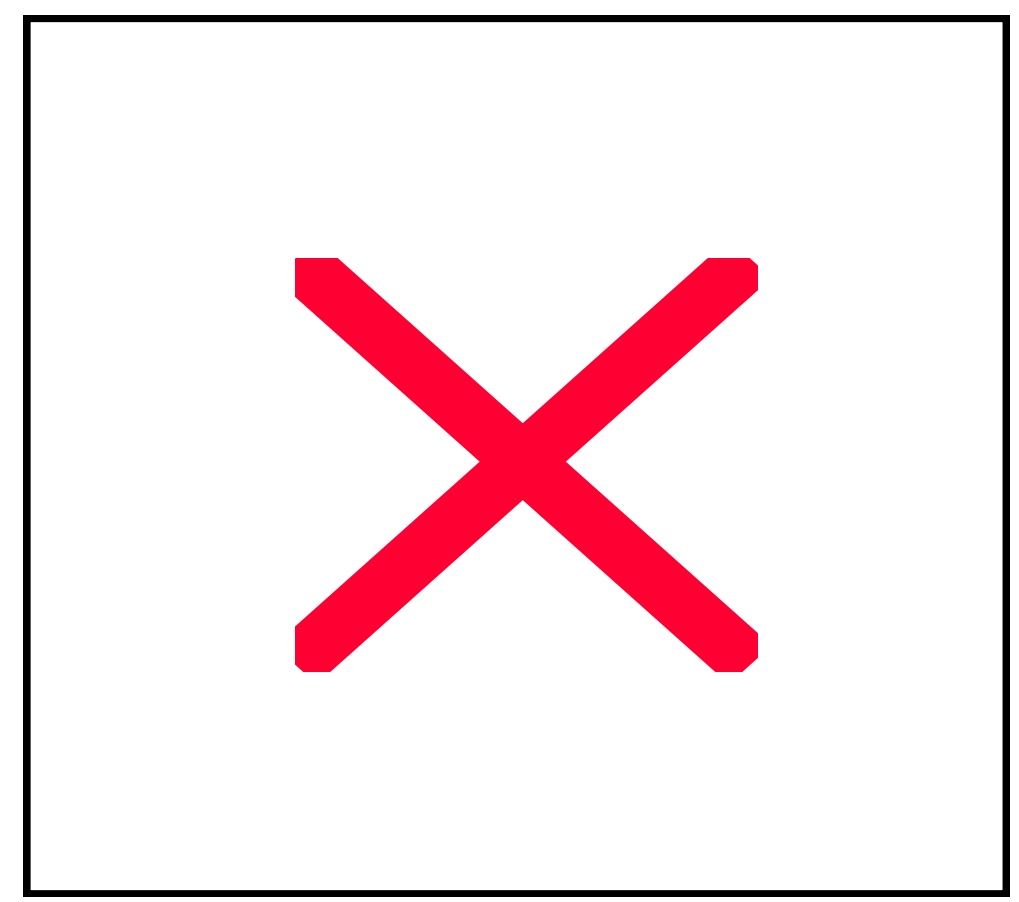

Figure 4: Application Integration Through Web Services

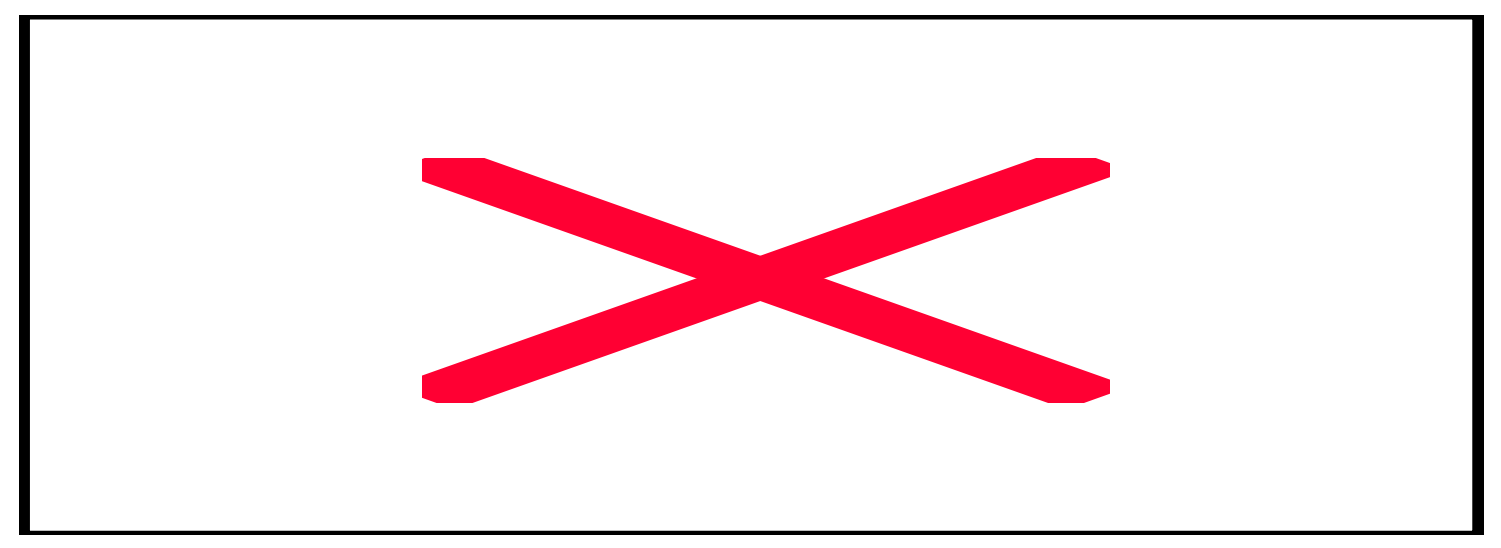

\subsection{Conclusion}

Pressure to reduce the amount of time required to originate a mortgage may lead many in the appraisal industry to adopt technological innovations. This paper focused on four such innovations, intelligent agents, XML, automated valuation models (AVMs), and web services. AVMs give the appraiser a powerful tool to help automate the appraisal process. AVMs are not designed to replace the appraiser only to help them render an estimate of value in a more efficient and effective manner. AVMs rely on statistics, neural networks, and expert systems. This paper discussed the issues surrounding neural networks and their application to real estate appraisal. Expert systems were also discussed and how they could be applied to the appraisal process. One issue that impacts the successful 
implementation of both a neural network and an expert system is the need for a large data set. Intelligent agents are another technological innovation discussed in this paper. Intelligent agents were introduced to show that creating, maintaining, and verifying data in a database can now be enhanced with the use of technology. Extensible markup language or XML was introduced to propose how the effectiveness of an intelligent agent could be increased. A sample query of residential property was utilized to show the power of XML is structuring data. An additional advantage of using XML for formatting data is that it forms the basis of web services - a recent phenomenon on the Internet. This paper also presented a brief overview of technologies behind web services and suggested use of AVM-based application and its exposure to the Internet through the use of web services.

\section{References}

1. [BAR 92] Barney, D.K., Graves, O.F., Johnson, J.D., "The Farmers Home Administration and Farm Debt Failure Prediction", Journal of Accounting and Public Policy, January 1999, p. 1-41.

2. [BRA 99] Bradshaw, J.M., Greaves, M., Holmback, H., Karygiannis, T., Jansen, W., Silverman, B.G., Suri, N., Wong, A., "Agents for the Masses?”, IEEE Intelligent Systems, March/April 1999, p. 53-63.

3. [CHI 00] Chiu, C., "Re-Engineering Information Systems with XML", Information Systems Management, Fall 2000, p. 40-54.

4. [DO 92] Do. A.Q., Grudnitski, G., "A Neural Network Approach to Residential Property Appraisal”, The Real Estate Appraiser, 1992, p. 38-45.

5. [DOR 94] Dorsey, R.E., Johnson, J.D., Mayer, W., "A Genetic Algorithm For the Training of Feedforward Neural Networks", Advances in Artificial Intelligence in Economics, Finance, and Management, 1994, p. 93-111.

6. [DOR 95] Dorsey, R.E. Mayer, W., "Genetic Algorithms for Estimation Problems With Multiple Optima, Nondifferentiability, and other Irregular Features", Journal of Business \& Economic Statistics, 1995, p. 5366.

7. [EVA 92] Evans, A., James, H., Collins, A., "Artificial Neural Networks: An Application to Residential Valuation in the UK", Journal of Property Valuation and Investment, 1992, 11, p. 195-204.

8. [GLU 99] Glushko, R.J., Tenenbaum, J.M., \& Meltzer, B., "An XML Framework for Agent Based ECommerce", Communications of the ACM, March 1999, 42 no. 3, p. 106-114.

9. [HAN 99] Handler, J., "Making Sense out of Agents", IEEE Intelligent Systems, March/April 1999, p. 3237.

10. [HAY 99] Hayes, C.C., "Agents in a Nutshell - A Very Brief Introduction", IEEE Transactions on Knowledge and Data Engineering, January/February 1999, 11 no. 1, p. 127-132.

11. [HOL 92] Holland, J.H., "Genetic Algorithms", Scientific America, July 1992, p. 66-72.

12. [KUS 99] Kushmerick, N., "Gleaning the Web", IEEE Intelligent Systems, March/April 1999, p. 20-22.

13. [MAE 99] Maes, P., Guttman, R.H., Moukas, A.G., "Agents that Buy and Sell", Communications of the ACM, March 1999, 42 no. 3, p. 81-91.

14. [SHO 03] Shohoud, Y., "Real World XML Web Services," Pearson Education, Inc.

15. [TAY 91] Tay, D., Ho, D., "Artificial Intelligence and the Mass Appraisal of Residential Apartments", Journal of Property Valuation and Investment, 1991, 10, p. 525-540.

16. [TOS 97] Tosh, D.S., Rayburn, W.B., Uniform Standards of Professional Appraisal Practice: Applying the Standards, 1997, Fourth Edition. Dearborn Press.

17. [VAN 00] Van den Hoven, J., "XML - Rosetta Stone for Data", Information Systems Management, Fall 2000, p. 55-58.

18. [WOR 95] Worzala, E., Lenk, M., Silva, A., "An Exploration of Neural Networks and Its Application to Real Estate Valuation", The Journal of Real Estate Research, 1995, 10 no. 2, 185-201. 